\title{
Strain Monitoring of Widening Cement Concrete Pavement Subjected to Differential Settlement of Foundation
}

\author{
Xiaolin Weng, Yongtao Nie, and Junyuan Lu \\ Key Laboratory for Special Area Highway Engineering of Ministry of Education, Chang'an University, Xian 710064, China \\ Correspondence should be addressed to Xiaolin Weng; 49768532@qq.com
}

Received 13 May 2015; Revised 3 August 2015; Accepted 13 August 2015

Academic Editor: Romeo Bernini

Copyright (C) 2015 Xiaolin Weng et al. This is an open access article distributed under the Creative Commons Attribution License, which permits unrestricted use, distribution, and reproduction in any medium, provided the original work is properly cited.

\begin{abstract}
This paper describes the use of a sensor based on a fiber Bragg grating (FBG), which is capable of reducing strain transfer errors, to measure the strain features within the top and bottom layers of a cement concrete pavement subject to different degrees of foundation settlement. Based on measured data, the characteristics of the distribution and the variation in the structure strain were analyzed in detail. By comparing the measured results with those obtained by means of finite element analysis, the proposed FBGbased sensor assembly was found to be capable of effectively monitoring the strain distribution in a cement concrete pavement, thus proving that the improved optical fiber grating strain sensor is a very promising solution for road pavement structural health monitoring.
\end{abstract}

\section{Introduction}

Assessing the condition and monitoring the health of structures constitute an important part of civil engineering during both construction and operation. Traditional structural strain monitoring techniques are not suitable for structures subject to complex or multi-interruption conditions [1-3]. The main advantages of optical fiber sensors over conventional monitoring techniques include their immunity to electromagnetic interference, their small size, and lightweight construction, which can overcome the limitations $[4,5]$. Optical fiber technology has been widely used for structure monitoring in civil engineering. Klar et al. [6] used optimization procedures to evaluate the ground displacement parameters using this technology, and the reliability of this new technology was verified from the obtained field test data. Recently, Weng et al. [7] reported on the monitoring results of a model test of an asphalt concrete pavement instrumented with embedded FBG strain sensors. Zhou et al. [8] introduced an optical fiber grating-based sensor assembly, which can measure 3D strain in large-scale structures. Recently, an increasing number of highway projects are being implemented with a goal of widening or reconstructing old roads. However, many road widening projects quickly saw the appearance of many longitudinal cracks, soon after the completion of construction [9-11]. Therefore, research into road widening projects has very practical significance. To date, this subject has given rise to the successful implementation of many research programs. Qinlong et al. [12] developed tests for a widened subgrade. For these tests, they employed a layer of soluble chemical fertilizer below the embankment fill, which was then dissolved in the ground water. In this way, they simulated differential settlement. By employing a large centrifuge tester along with PLAXIS, Hortnz-Pedersen and Broers [13] analyzed the deformation characteristics of subgrade widening on a soft foundation. Allersma et al. [14] analyzed the destabilization of the widened subgrade and evaluated two methods of embankment-fill placement by using the same means. It should be noted, however, that all these study programs involve non-system-model tests and lack prototype observation data. Therefore, the results of these studies cannot provide sufficient data support for the pavement structure failure mechanism or the deformation model based on uneven settlement of the foundation. In addition, it is very difficult to establish a complete numerical analysis model for the widening of a road and the analysis for the deformation distribution and strain condition, mainly because these factors are so complicated. Due to the limitations on the size of 
the model box, a conventional model test cannot accurately represent the prototype in terms of mechanical properties. Therefore, it is necessary to undertake an influence mechanism study on the widening of cement concrete pavement under different degrees of foundation settlement based on the Changan University Foundation settlement platform. This paper mainly describes the use of an FBG sensor to monitor the strain features of a concrete pavement structure in fullsize model testing. The paper is divided into two parts. In part 1, we present the physical background to Bragg grating sensors and their application to full-size model tests. In part 2 , the results of experiments are presented and compared with those of finite-elements method (FEM) simulations.

\section{The Principle of Bragg Grating Technology}

Fiber grating is a kind of sensing technology based on Bragg grating reflecting light wave with specific wavelength. When a broadband light source is injected into the optical fiber, the FGB will reflect a part of narrow spectrum light with specific wavelength and then generate a certain reflection spectrum. The relationship is given by [15]

$$
\lambda_{B}=2 n_{\mathrm{eff}} \Lambda \text {, }
$$

where $\lambda_{B}$ is the Bragg wavelength, typically $1510 \mathrm{~nm}$ to $1590 \mathrm{~nm} ; n_{\text {eff }}$ is the effective core index of refraction; and $\Lambda$ is the period of the index modulation.

The center wavelength of the reflected light has a specific relationship with the optical fiber strain and temperature. The relationship is given by [16]

$$
\frac{\Delta \lambda_{B}}{\lambda_{B 0}}=c_{\varepsilon} \varepsilon+c_{T} \Delta T,
$$

where $\Delta \lambda_{B}$ is the variation in the Bragg wavelength due to changes of external conditions; $\lambda_{B 0}$ is the original Bragg wavelength; and $c_{\varepsilon}$ and $c_{T}$ are the calibration coefficients of strain and temperature.

As shown in Figure 1, FBG sensor is also very sensitive to temperature change, so the temperature-compensation problem must be solved. During the test, by adding another temperature sensor, the temperature $\Delta T$ of the same field is measured, and then the test strain values would be modified.

Although the FBG sensor offers the advantages of high accuracy and durability, due to the unfriendliness of construction environments and the unique features of civil engineering, FGB sensing technology is limited in terms of the instrument layout, cable protection, and data analysis, which greatly affects the promotion and application of the monitoring techniques. Specifically, the main application difficulty originates in two areas. First one is the accurate installation and on-site protection of the fiber optic cable transmission grating. To allow the fiber grating to reflect the deformation, stress, and other physical values of the structure, it is necessary to ensure synchronization deformation between the fiber grating and the object. During the instrument-laying and concrete-pouring processes, due to the harsh construction environment, the fiber grating and

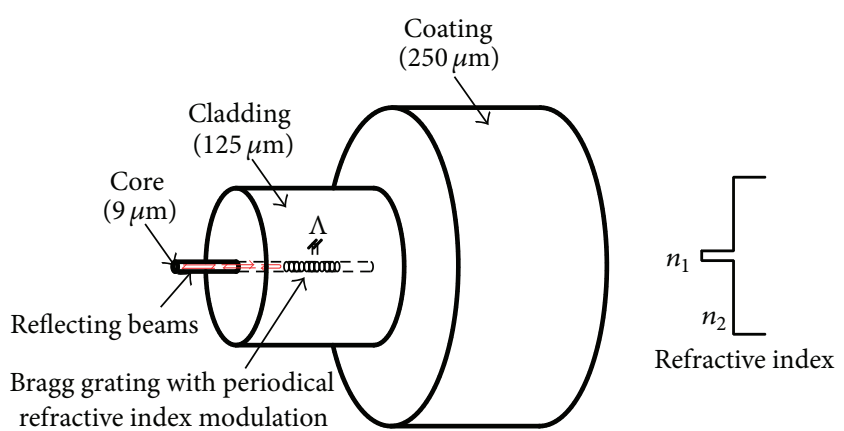

FIGURE 1: Operating principle of an FBG sensor.

transmission cable are vulnerable to damage. Therefore, it is very important to implement effective protection measures. In addition, to accurately obtain the strain in the pavement structure and its variation with temperature, fiber grating readings must isolate strain and temperature in the process of long-term monitoring. Failure to implement temperature compensation will, for an ambient temperature change of $1^{\circ} \mathrm{C}$, produce a strain error of about $9 \mu \varepsilon$. Thus, if the issues are not handled appropriately, the monitoring data will not reflect the true status of the site.

\section{Model Testing}

3.1. Foundation Settlement Simulation System. The main source of the settlement of a widened roadbed is the differential foundation settlement. The lift system of the model test simulation platform consists of hydraulic jacks, which can simulate the settling values and the sedimentation rate of the foundations. The multiple rig panels of the test platform are assembled into a "ground surface" with manual control, while translational and rotational movements are achieved using the activities panel under the control of the lifting system, which can be approximated by simulating the actual surface foundation settlement. The foundation settlement system is shown in Figure 2. During the test, a different point foundation subgrade settlement value was simulated by adjusting the degree of lifting at the different jack positions. The maximum settlement of the foundation $\left(S_{2}\right)$ was set to the shoulder position of the new subgrade, while the minimum value for foundation $S_{1}$ was set to the midline of the old subgrade, with $S=S_{2}-S_{1}$ representing the difference between the two positions. During the test, different deformation curves were produced by the jacking system. The $S$ values of test were set to between $2 \mathrm{~cm}$ and $10 \mathrm{~cm}$ by the control system, to analyze the strain response of the cement concrete pavement to foundation settlement.

3.2. Bonding Methodology of FBG Sensors. However, being thin, the optical fiber itself has only limited tension and shear resistance; therefore, the application of effective sensor protection measures for the experiment is very important. To protect the optical FBG sensor, a metal tube is commonly used for the package, as well as the embedded concrete strain gauge. Therefore, tube-packaged fiber-grating sensors were 

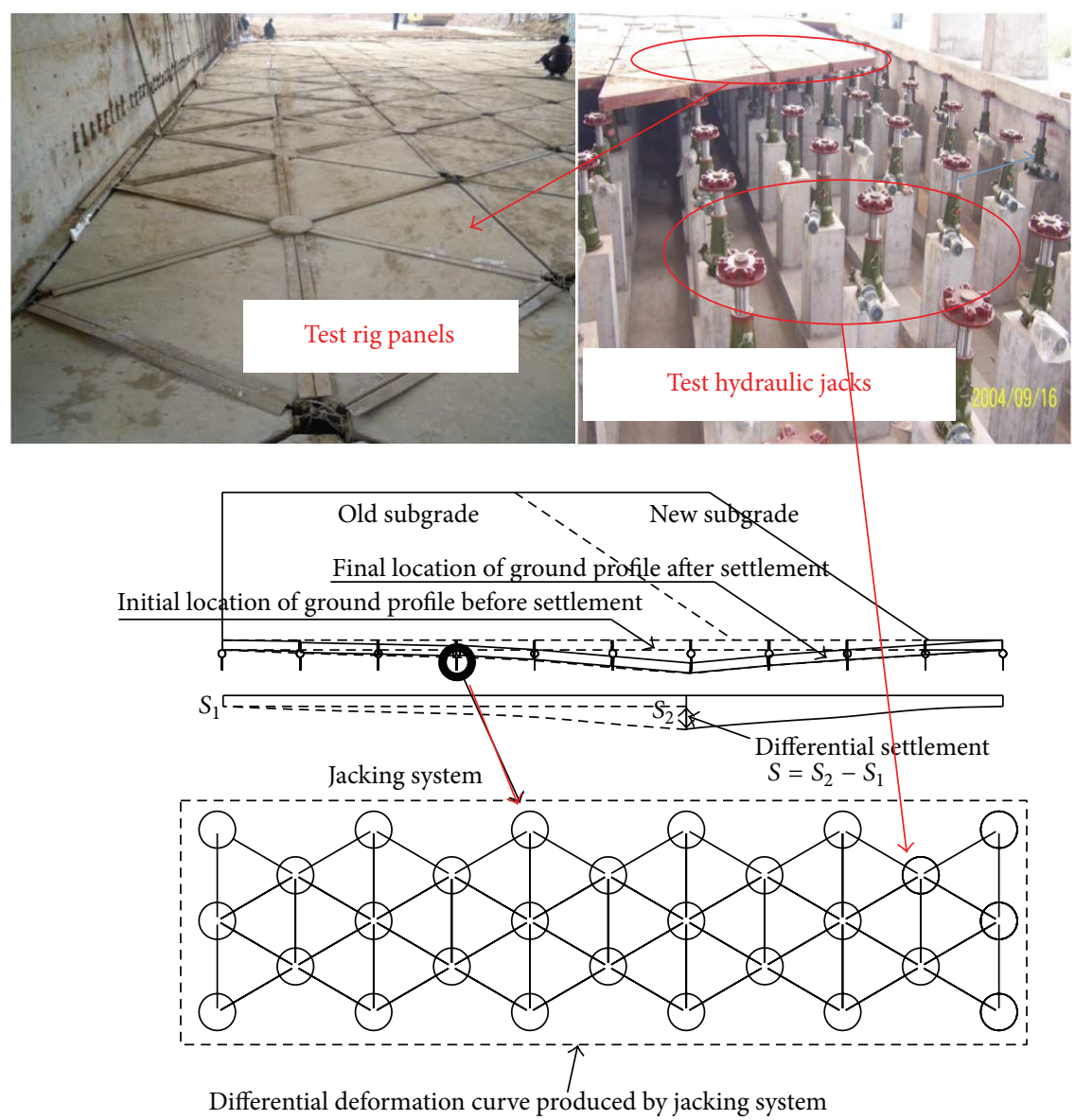

FIGURE 2: Settlement profile applied to road embankment.

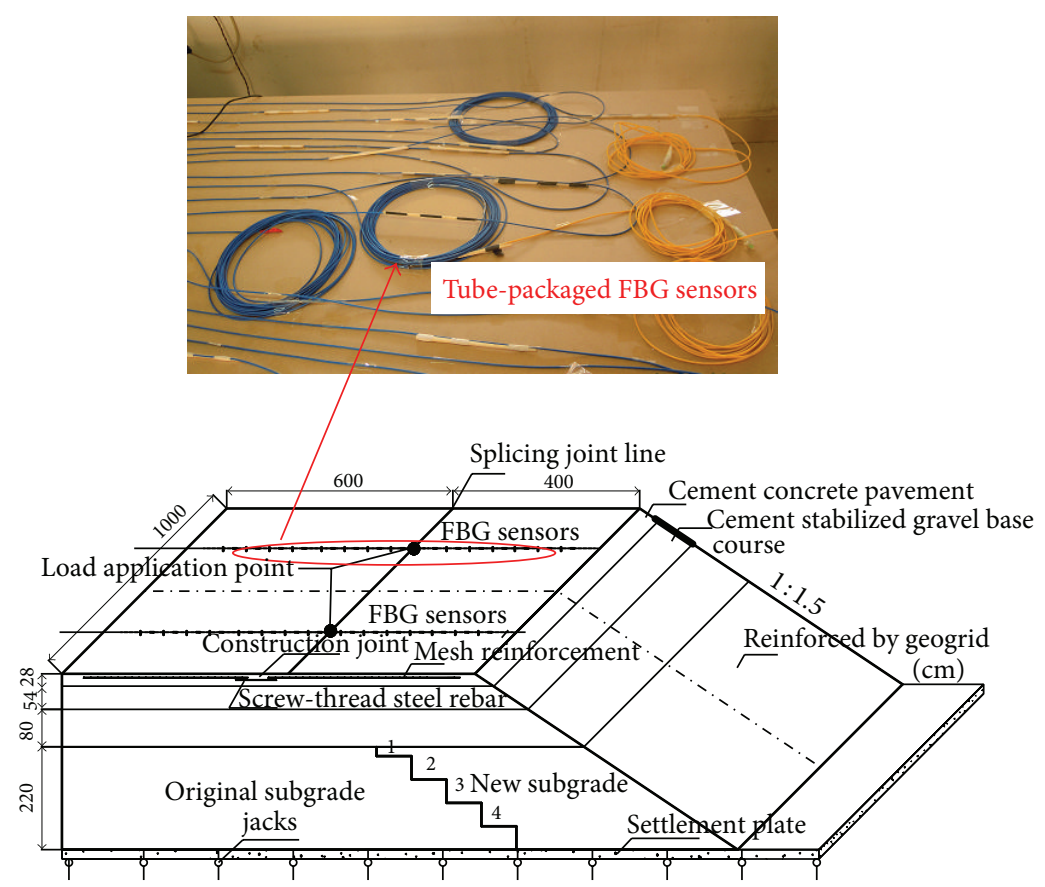

FIGURE 3: FBG strain-sensing system. 


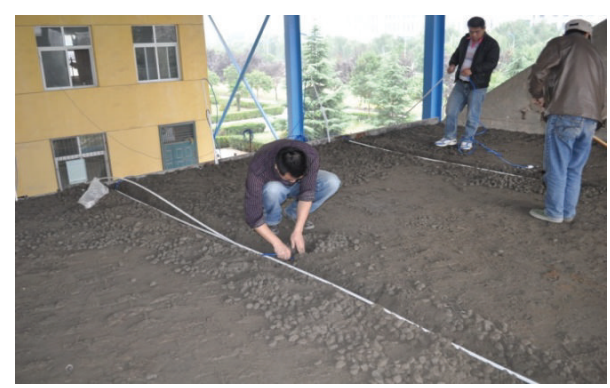

(a) Embedded FBG temperature sensor

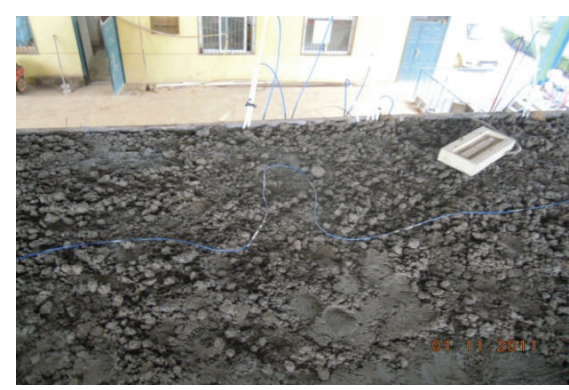

(b) Location of sensor network

FIGURE 4: FBG sensor-network construction.

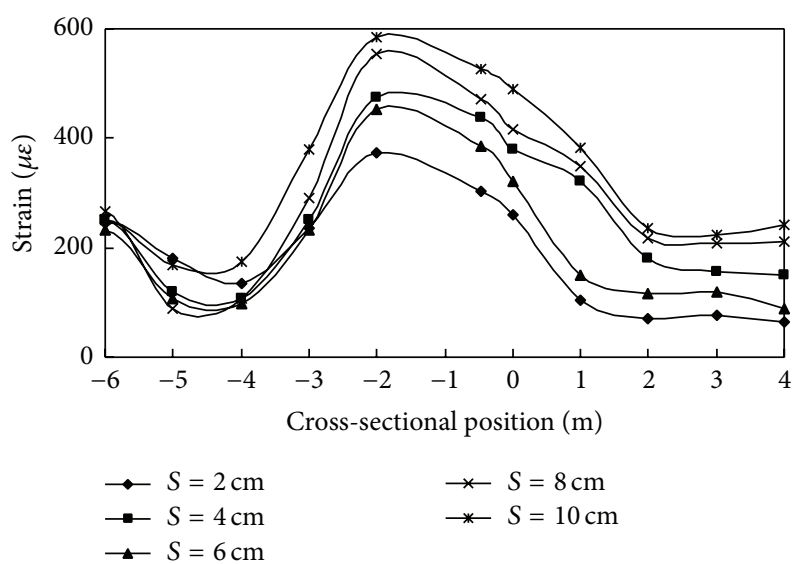

(a) Top of concrete pavement (with geogrid)

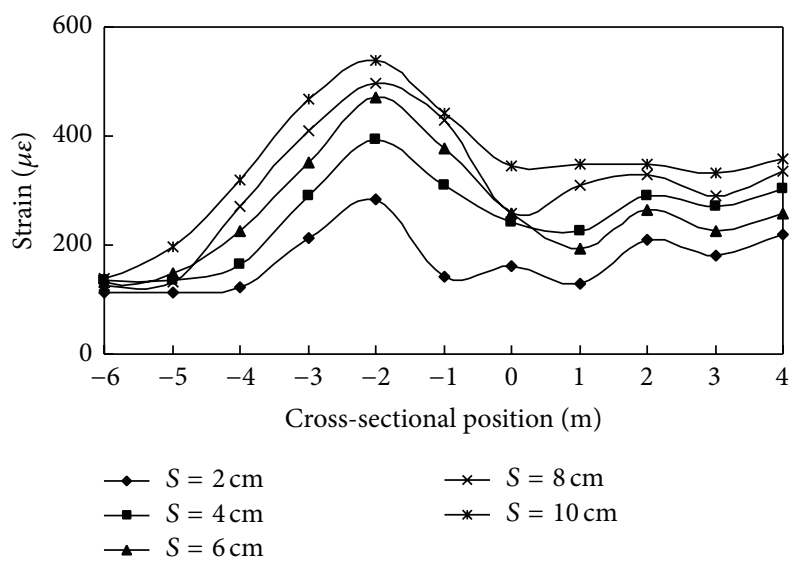

(c) Top of concrete pavement (no geogrid)

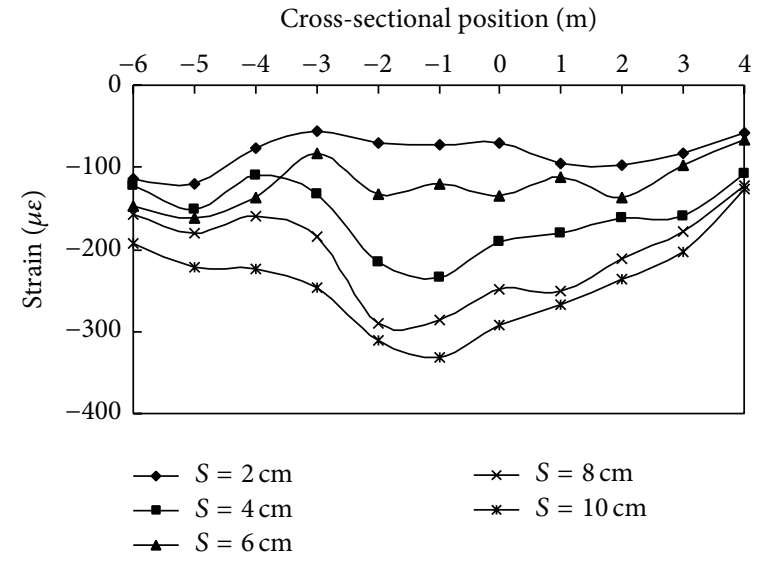

(b) Bottom of concrete pavement (with geogrid)

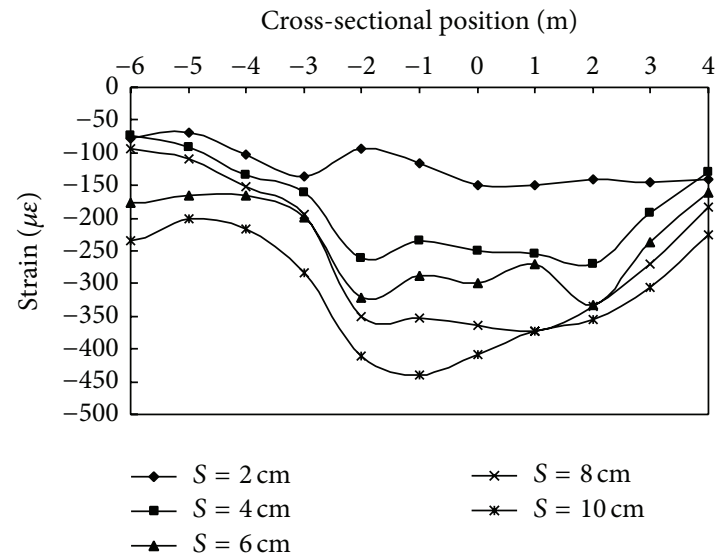

(d) Bottom of concrete pavement (no geogrid)

FIGURE 5: Variation of strain in the pavement as measured with FBG sensors.

independently designed and produced for the model test. Before the fiber grating was encapsulated into a pipe, the bare grating was wrapped in four layers of thermal plastic pipe, as shown in Figure 3. This packaging technology ensures the survival rate of the fiber grating sensor. An old pavement with a half-width of $6 \mathrm{~m}$ was designed to be widened to $10 \mathrm{~m}$.
The transverse length of the embankment was $10 \mathrm{~m}$. To evaluate the contribution of geogrids embedded in the embankment to the enhancement of the performance of the pavement, half of the embankment was reinforced with two layers of geogrids. Both the top and the bottom of the cement concrete layers were instrumented with forty FBG 


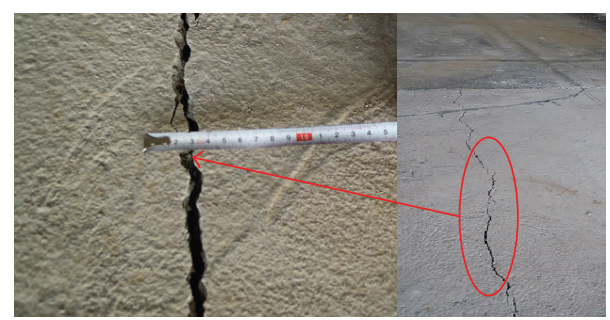

FIgURE 6: Crack observed in pavement after test.

strain sensors, which were divided into four sets, two sets for the reinforced embankment and another two sets for the nonreinforced embankment. Each set of sensors was instrumented on the transverse midline of the top and bottom concrete layers.

During the experiment, these sensors can basically perform real-time monitoring of the strain in the concrete pavement structure, with only a few failings by the end of the test, due to excessive pavement cracking. To enable temperature compensation, $5 \mathrm{~mm}$ metal tube was used to package the fiber grating and thus fabricate an embedded fiber Bragg grating thermometer. The fiber length has some redundancy in the metal pipe, to ensure that the grating is always in a relaxed state while in use and not affected by external strain.

Photographs of the construction of the FBG sensors are shown in Figure 4. Temperature data is collected and then, using a specific formula, the testing strain value is corrected, as follows:

$$
\Delta \varepsilon=\frac{1}{c_{\varepsilon}}\left(\frac{\Delta \lambda_{B}}{\lambda_{B}}-c_{T} \Delta T\right) .
$$

\section{Analysis of Test Results}

Figure 5 illustrates the relationship between the strain in the concrete cement pavement and the differential settlement of the foundation. From Figure 5, it can be seen that the top layer of the cement concrete pavement structure produces tensile strain under the influence of the differential settlement of the ground conditions. The strain gradually increases with the increase in $S$. A relatively steady stage with small magnitude is observed before $S$ reaches $8 \mathrm{~cm}$. When $S$ surpasses $8 \mathrm{~cm}$, however, the strain begins to increase more rapidly. At this point, due to the additional stress caused by considerable differential settlement of the ground, structural failure develops in the pavement and cracking occurs on the surface, as shown in Figure 6. A comparison between Figures 5(a) and 5(c) reveals that, due to the application of a geogrid, the strain in Figure 5(a) is relatively low with a maximum value of 586 versus 594 in Figure 5(c) to which no geogrid is applied. This is because the geogrid enhances the consolidation effect of the soil body and helps relieve the additional strain in the pavement as well as the differential settlement of the ground. Instead, from Figures 5(b) and 5(d), it can be seen that, with the increase in the differential settlement of the ground, the bottom of the pavement exhibits a variation in

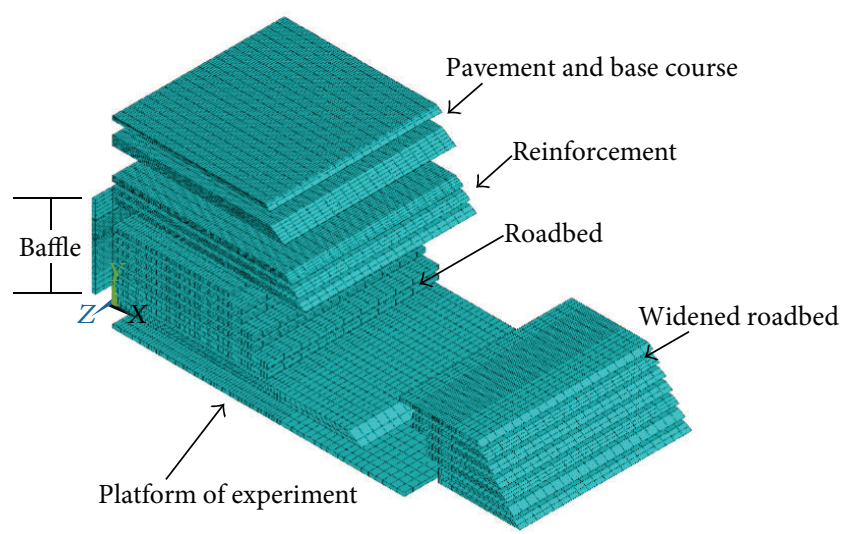

FIgURE 7: FEA model.

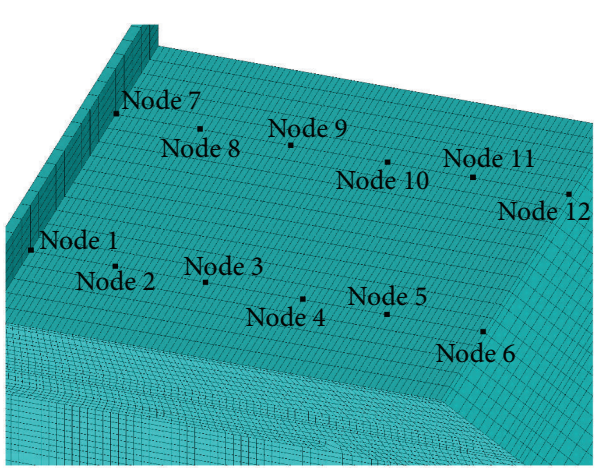

FIGURE 8: Pavement strain inspection nodes.

the tensile strain, which exhibits similar strain characteristics to those of Figures 5(a) and 5(c). Basically, therefore, the strain variation law is compatible with the differential settlement of the foundation. Because of the differential settlement of the natural ground and the nonuniformity of both the compressive deformation of the subgrade and the accumulative deformation of the ground, the new subgrade of a road widening project, especially when placed on a soft foundation, is subjected to surface differential settlement that may possibly cause hollows under the pavement. Such hollows are most likely to form along the center line of a new subgrade. In other places, such as around the splicing joint range (taking the midline as the benchmark, from $-2 \mathrm{~m}$ on the left side to $1 \mathrm{~m}$ on the right), the settlement or deformation of the excessive amount is unlikely to occur due to the load limits being exceeded.

\section{Comparative Analysis through Finite Element Analysis}

The objective of the application of finite element analysis (FEA) was to verify the reliability of the FBG sensors in the large-scale model tests. The mathematical modeling was devised on the basis of large-scale tests, as shown in Figure 7. The analysis process mainly considered the differential settlement of the foundation, which was increased in steps of $1 \mathrm{~cm}$, for a total of 24 different working conditions. 


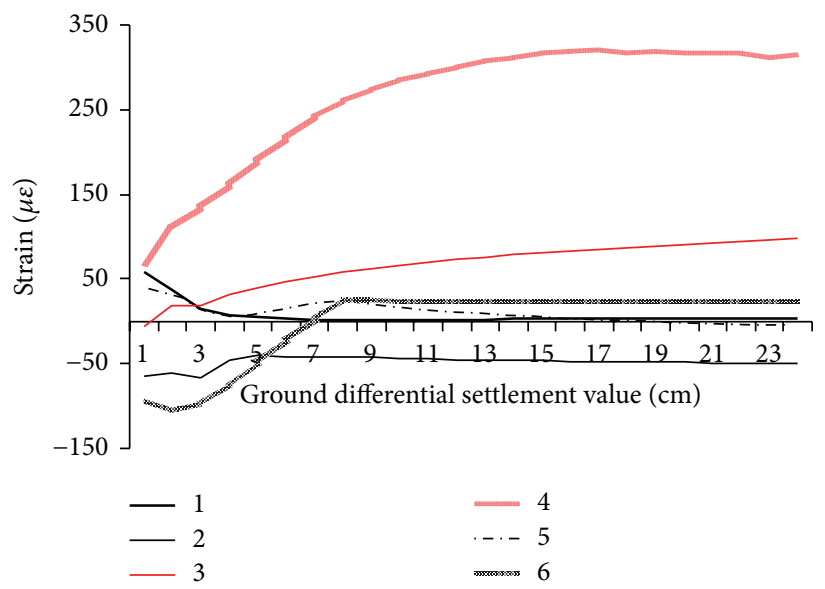

(a) Top of concrete pavement (with geogrid)

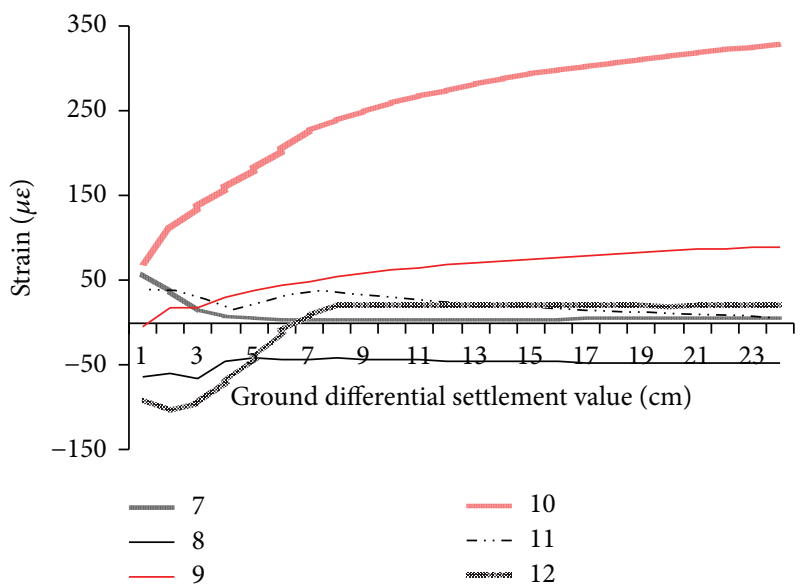

(b) Top of concrete pavement (no geogrid)

FIGURE 9: Variation curves for strain in the pavement structures as determined by FEA.

As shown in Figure 8, the strain data for Nodes 1 to 12 were observed, so as to obtain the strain curves for the concrete pavement subjected to differential settlement of the foundation (Figure 9). From Figure 9, we can see that points away from the center line within a range of $3.55 \mathrm{~m}$ to $9.19 \mathrm{~m}$ of the surface layer bear the majority of the additional tensile strain, while the other positions bear the compressive strain. Between Node 3 and Node 4 regions, the subgrade zone up to $5.51 \mathrm{~m}$ from the center line bears the largest tensile strain, implying that the top surface of the pavement, near the splicing region, incurs the maximum additional tensile stress as a result of the differential foundation settlement, where there is an unfavorable position structure layer as well as a mechanical control area. Comparing Figure 9(a) with Figure 9(b), laying a geogrid on both the new and old roadbed splicing regions can greatly alleviate the additional strain arising from the differential settlement in the foundation. When the differential settlement value reaches $10 \mathrm{~cm}$ to $13 \mathrm{~cm}$, the strain rate at Node 4 becomes smaller and then gradually stabilizes. The tension strain at Node 10, which is located at a point at which a geogrid is not laid down, exhibits a continuation in the upward trend. Through a comprehensive analysis, the results of the finite element numerical analysis and those of the FBG sensor monitoring were found to be broadly consistent.

\section{Conclusions}

Fiber optic sensors were used in a large-scale-model test of a road pavement layer. As a result, the cement concrete pavement layer strain characteristics subject to differential foundations settlement could be effectively monitored and evaluated. To effectively protect a sensor in hostile construction environments and thus attain reasonable monitoring data, a protection system based on a tube-package was developed and implemented. A comparison between the measured and FEA results showed that both results exhibit the same variations and trends, which show that the improved optical fiber grating strain sensor constitutes a very promising solution for application to road pavement structural health monitoring, as well as other civil structures.

\section{Conflict of Interests}

The authors declare that there is no conflict of interests regarding the publication of this paper.

\section{Acknowledgment}

This work was supported by the National Natural Science Foundation of China (Grants nos. 51008032 and 51378004).

\section{References}

[1] Y. B. Lin, C. L. Pan, Y. H. Kuo, K. C. Chang, and J. C. Chern, "Online monitoring of highway bridge construction using fiber Bragg grating sensors," Smart Materials and Structures, vol. 14, no. 5, pp. 1075-1082, 2005.

[2] T. Matsumoto, P. Kitiyodom, H. Matsui, and Y. Katsuzaki, "Monitoring of load distribution of the piles of a bridge during and after construction," Soils and Foundations, vol. 44, no. 4, pp. 109-117, 2004.

[3] J. M. López-Higuera, C. J. Misas, A. Q. Incera, and J. Echevarría Cuenca, "Fiber optic civil structure monitoring system," Optical Engineering, vol. 44, no. 4, 2005.

[4] S.-C. Her and C.-Y. Tsai, "Strain measurement of fiber optic sensor surface bonding on host material," Transactions of Nonferrous Metals Society of China, vol. 19, supplement 1, pp. s143-s149, 2009.

[5] K. Kesavan, K. Ravisankar, S. Parivallal, P. Sreeshylam, and S. Sridhar, "Experimental studies on fiber optic sensors embedded in concrete," Measurement, vol. 43, no. 2, pp. 157-163, 2010.

[6] A. Klar, I. Dromy, and R. Linker, "Monitoring tunneling induced ground displacements using distributed fiber-optic sensing," Tunnelling and Underground Space Technology, vol. 40, pp. 141-150, 2014.

[7] X. Weng, H.-H. Zhu, J. Chen, D. Liang, B. Shi, and C.-C. Zhang, "Experimental investigation of pavement behavior after 
embankment widening using a fiber optic sensor network," Structural Health Monitoring, vol. 14, no. 1, pp. 46-56, 2015.

[8] Z. Zhou, W. Liu, Y. Huang et al., "Optical fiber Bragg grating sensor assembly for 3D strain monitoring and its case study in highway pavement," Mechanical Systems and Signal Processing, vol. 28, pp. 36-49, 2012.

[9] Y. Chen, L. V. Yue, and Z. Zhang, "The cause of longitudinal pavement fissures and preventions on highway road-widening engineering," East China Highway, vol. 40, no. 1, pp. 38-41, 2003 (Chinese).

[10] W. Xiaolin, L. Lintao, and Z. Liujun, "Model experimental research on wetting damage mechanism of widening loess roadbed," Chinese Journal of Rock Mechanics and Engineering, vol. 29, no. 5, pp. 1075-1081, 2010 (Chinese).

[11] H. Jie and A. Ken, "Use of geogrid-reinforced and pilesupported earth structures," in Proceeding of the International Deep Foundation Congress, pp. 668-679, ASCE, Orlando, Fla, USA, February 2002.

[12] H. Qinlong, L. Jianming, and T. Boming, "Experimental research on incoordinate deformation between existing subgrade and the widening one," Journal of Highway and Transportation Research and Development, vol. 21, no. 12, pp. 18-21, 2004 (Chinese).

[13] A. G. I. Hortnxs-Pedersen and H. Broers, "The behaviour of soft subsoil during construction of an embankment and its widening," in Proceedings of the International Conference Centrifuge, pp. 567-574, A. A. Balkema, Rotterdam, The Netherlands, 1994.

[14] H. G. B. Allersma, L. Ravenswaay, and E. Vos, "Investigation of road widening on soft soil using a small centrifuge," Transportation Research Record, vol. 1462, pp. 47-53, 1994.

[15] W. W. Morey, G. Meltz, and W. H. G. Glenn, "Fiber optic bragg grating sensors," in Fiber Optic and Laser Sensors VII, vol. 1169 of Proceedings of SPIE, pp. 98-107, Boston, Mass, USA, 1989.

[16] A. D. Kersey, M. A. Davis, H. J. Patrick et al., "Fiber grating sensors," Journal of Lightwave Technology, vol. 15, no. 8, pp. 1442-1463, 1997. 

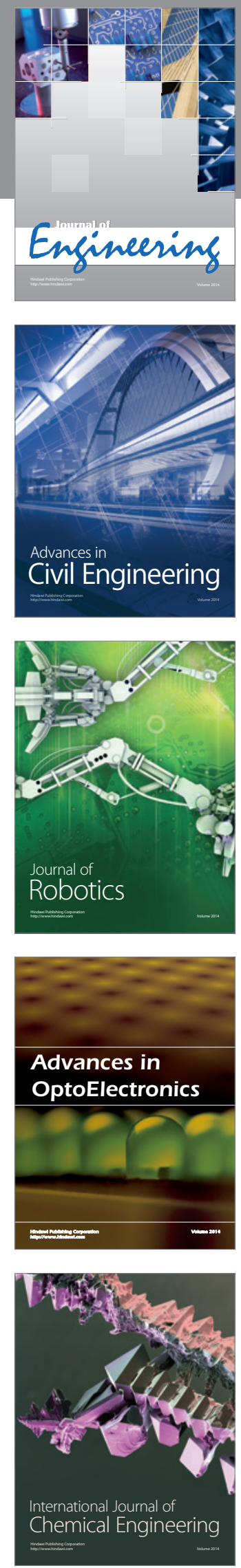

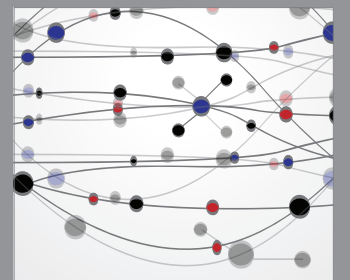

The Scientific World Journal
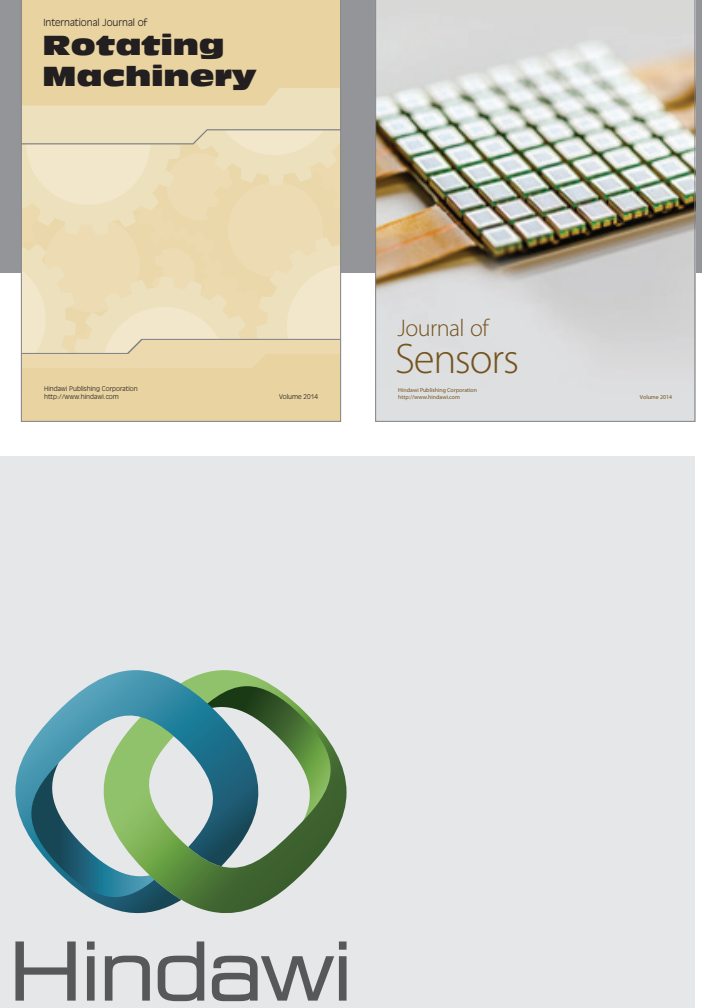

Submit your manuscripts at http://www.hindawi.com
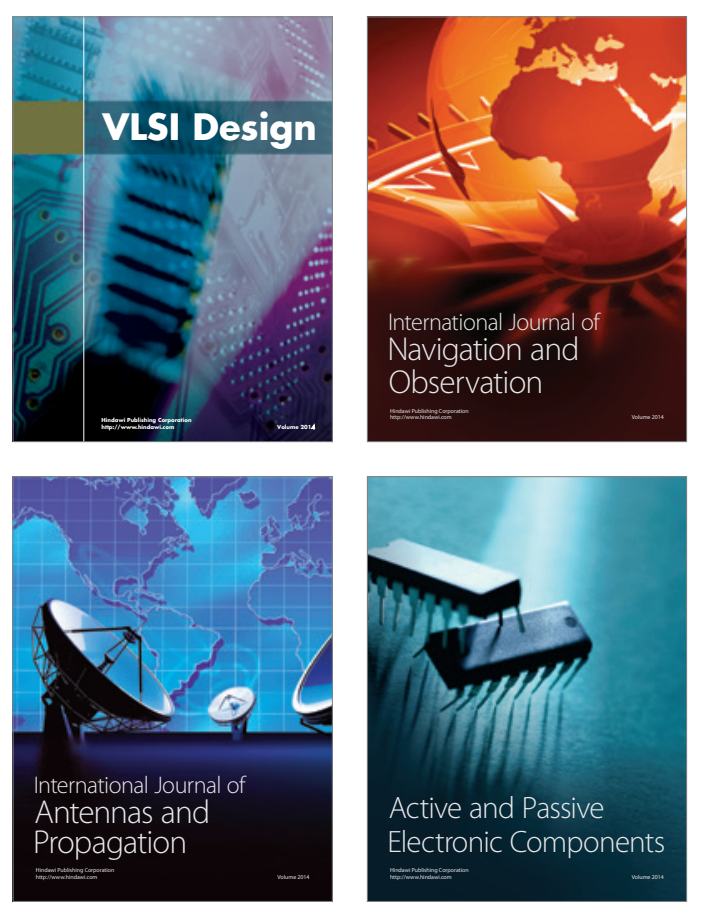
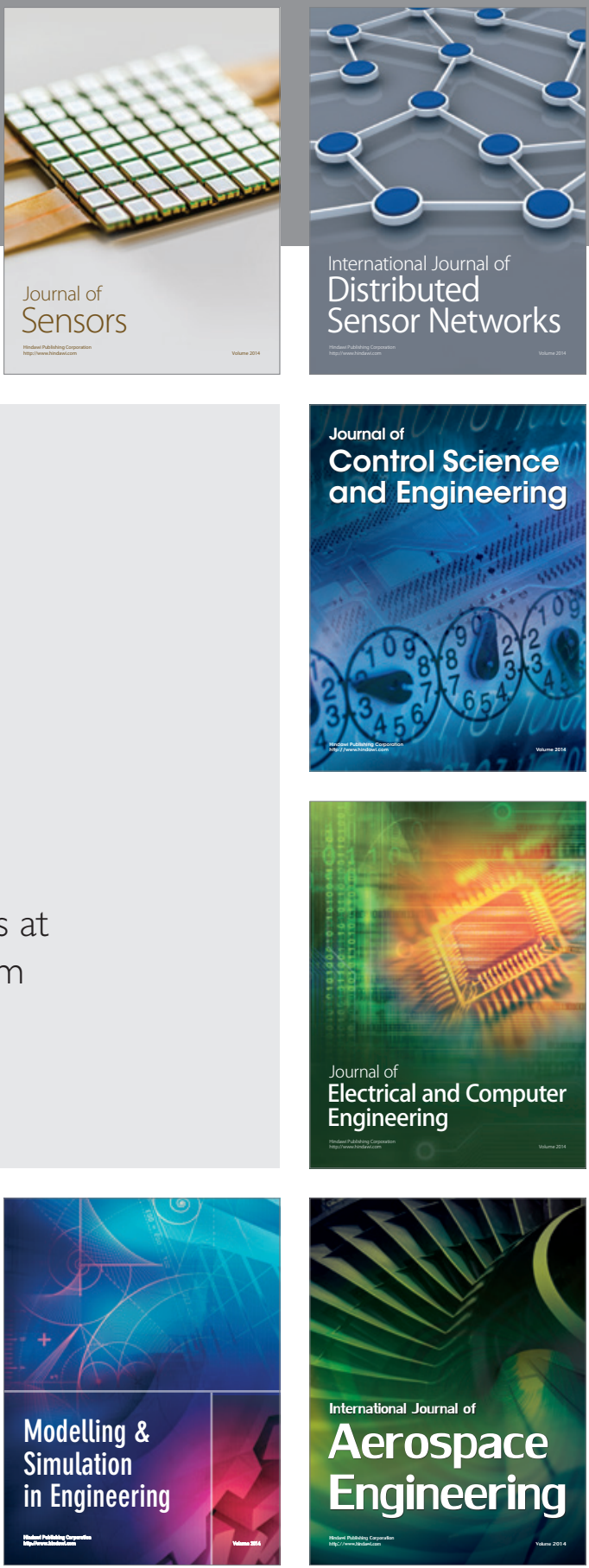

Journal of

Control Science

and Engineering
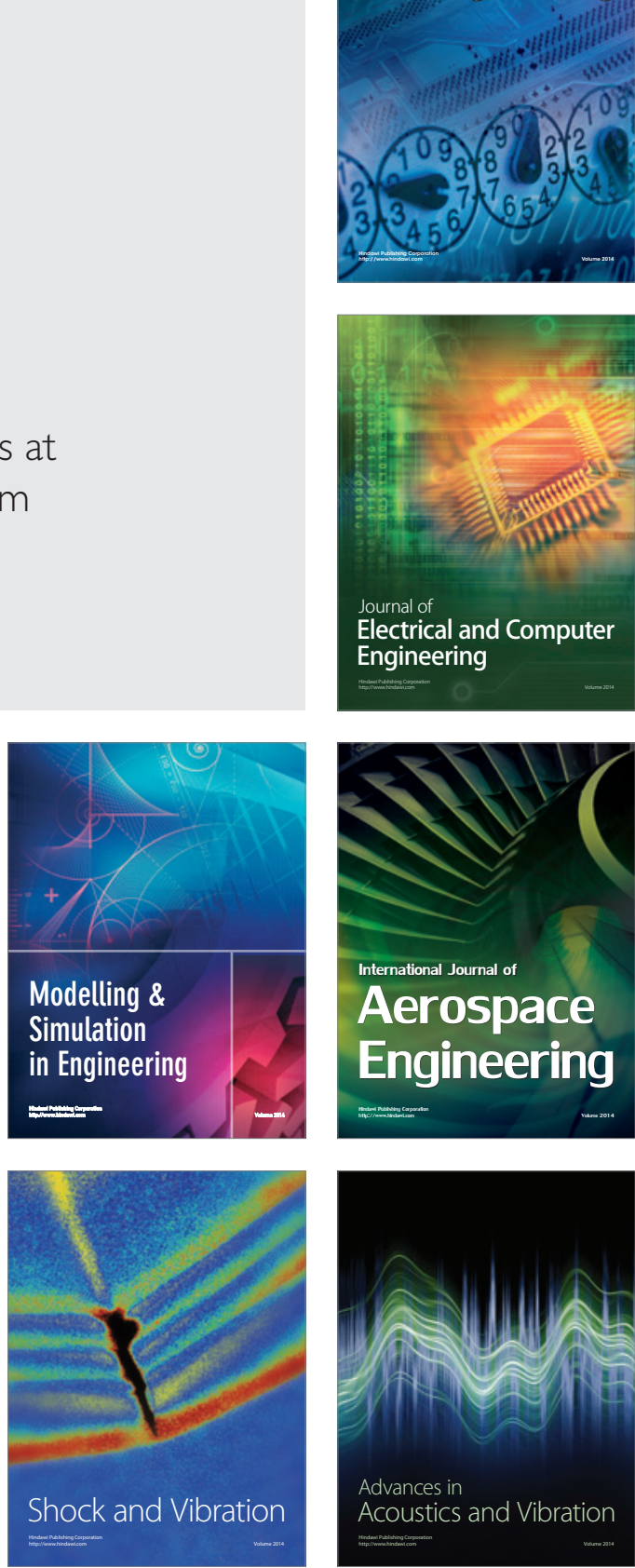